\title{
Pixel Classification Algorithms for Noise Removal and Signal Preservation in Low-Pass Filtering for Contrast Enhancement
}

\author{
Chunyan Wang and Sha Gong \\ Department of Electrical and Computer engineering, Concordia University, Montreal, Canada \\ 1455 de Maisonneuve West, Montreal, Canada H3G1M8 \\ emails: \{chunyan,sh_gon $\} @$ ece.concordia.ca
}

\begin{abstract}
With a view to obtaining a high quality contrast enhancement, low-pass filters are used to remove the noise generated in a high-gain histogram equalization process. To preserve signal variations, the LP operation applied to the pixels in non-homogeneous regions should have less smoothing strength than that in homogeneous regions. The pixel classification according to the gray level homogeneity is thus a critical part in the LP filtering. In this paper, two algorithms for pixel classification according to the gray level homogeneity of their regions are proposed. In each of them, image pixels are grouped in such a way that, in the same group, pixels in homogeneous regions can be easily distinguished from those in nonhomogeneous regions by a simple gradient thresholding, despite the complexity of signal gradient degradation in images. The two proposed classification algorithms are very simple, requiring very small quantity of computation. Their effectiveness has been proven by the simulation results.
\end{abstract}

Key words - Gray level homogeneity; high dynamic range (HDR); noise removal; signal preservation; low-pass filtering; high-pass filtering; gradient detection; contrast enhancement.

\section{INTRODUCTION}

Adaptive histogram equalization (AHE) algorithms, such as CLAHE [1], are widely used to enhance effectively image contrast. Most of AHE algorithms, however, result in undesirable noise that can be very pronounced, affecting the processing quality. There are two approaches to the noise reduction in the process of contrast enhancement. The first is to make the transformation function of histogram equalization more adaptive to local image signals. It can be done by modulating the "local" cumulative functions [2] or by adaptive clipping [3]. The other approach is to use low-pass filters to remove the noise generated by a histogram equalization process. Low-pass filters are used to reduce the blocking effect [4]. In [5], a transportation map regularization (TMR) filter is used for noise removal after CLAHE.

The involvement of low-pass filtering allows the enhancement of the image contrast and the reduction of the noise to be performed separately, and it is thus very promising. The scheme presented in [6] uses simple low-pass filter stages to achieve an effective noise removal while preserving signal variations. The successive filtering operations given by the cascaded stages provide different smoothing effects and a binary mask is used in each stage to expose/shield the pixels to make the filtering discriminative.
The processing quality of this scheme depends, however, on the quality of the classification of the pixels according to the homogeneity of their regions. In [6], some adjustments in the classification need to be done manually, which makes the quality control less evident.

The objective of the work presented in this paper is to develop algorithms of the pixel classification for the multistage low-pass filtering in the contrast enhancement scheme for a noise removal and signal preservation to achieve a highquality low-noise enhancement. The algorithms are designed to process varieties of low-contrast images in which the signal quality may be severely affected by gradient degradation, and to generate protection masks for the discriminative low-pass filtering in the contrast enhancement scheme.

\section{Proposed Algorithms}

Signal gradients of the pixels in a region can be used to detect the gray level homogeneity [7]. With a view to generating the masks controlling the multistage low-pass filtering in the contrast enhancement scheme shown in Fig. 1, high-pass operators are included to generate the signal gradients. The pixels in an image may be divided into two classes, namely the homogeneous and non-homogeneous ones, by a simple gradient thresholding under the condition of good-quality gradients. Unfortunately the signal gradients in an image requiring a contrast enhancement are usually of poor quality. Hence, the pixels should be grouped in such a way that in the same group, the pixel gradients are easily "distinguishable" so that a high-quality classification can be achieved by a simple thresholding in each group. In this section, the basic method of gradient threshold generation is presented and two different classification algorithms, each using this basic method but having its specific pixel grouping, are proposed.

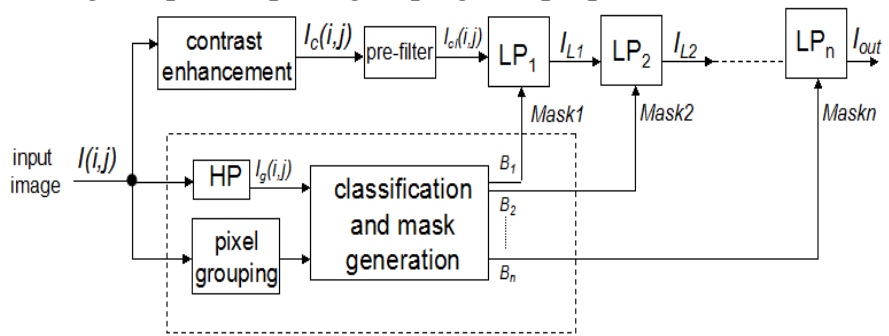

Fig. 1 Block diagram of the contrast enhancement in which a new procedure of pixel classification and mask generation, shown in the dashed frame, is incorporated. 


\section{A. Analysis of the gradient distribution and the threshold generation for pixel classifications}

Gradient values of the pixels in an image can be obtained by high-pass operations, such as SOBEL convolutions. Fig. 2 illustrates a typical distribution, or histogram $H\left(G_{r}\right)$, of the gradients $G_{r}$ obtained from such an operation. It is commonly known that the pixels in homogeneous regions are placed in the left part of the gradient distribution and those in nonhomogeneous regions in the right part. It may be difficult to determine the exact threshold dividing the pixels into the two groups. However for a practical purpose, one can define three gradient thresholds, $T h_{L}, T h_{H}$, and $T h_{M}$, in such a way that (i) all the pixels having their gradients smaller than $T h_{L}$ are classified as homogeneous pixels, (ii) those having their gradients greater than $T h_{H}$ as non-homogeneous ones, (iii) those having their gradients between $T h_{L}$ and $T h_{M}$ as nearhomogeneous ones, and (iv) the remaining pixels as near-nonhomogeneous ones.

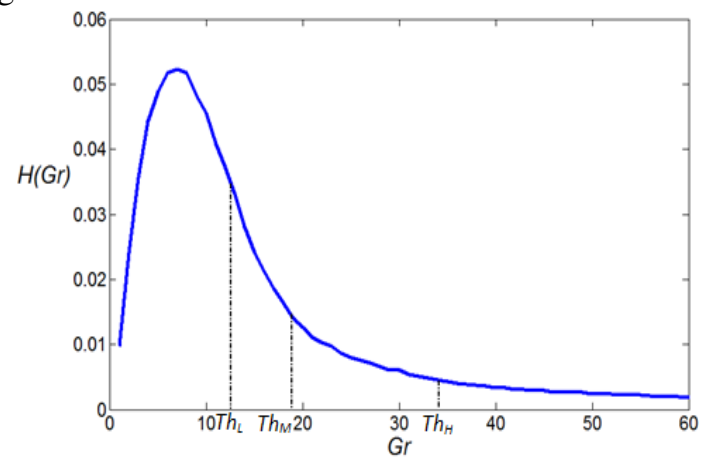

Fig. 2 Gradient distribution of the pixels of an image.

The gradient histogram presented in Fig. 2 illustrates that a good concentration of the pixels in homogeneous regions is found in the left side of the gradient distribution, but it reduces rapidly around the point of the maximum value of $\left|\frac{d H_{t} G_{2}}{d \sigma_{r}}\right|$, or $\frac{d^{2} H\left(G_{H}\right)}{d \sigma^{2}}=0$. The threshold $T h_{L}$ is found at this point. The threshold $T h_{H}$ is reasonably found at a point where the

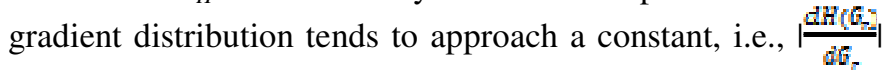
being at a level close to zero, such as 0.001 . The other threshold, $T h_{M}$, is between $T h_{L}$ and $T h_{H}$, at the point where $\frac{d^{2} P\left(Q_{x}^{2}\right.}{d \theta_{z}^{2}} \mid$ reaches its peak value. Thus, these threshold values can be easily found in the derivatives of the gradient distribution.

The above-described threshold generation is done by means of the gradient distribution analysis. It is used in the two algorithms for pixel classification in order to generate the masks to be used in the scheme shown in Fig. 1.

\section{B. Classification algorithm with pixel grouping based on gray level ranges}

This classification algorithm aims at low contrast images acquired in high dynamic range (HDR) scenes. The over-andunder exposure in the acquisition process may seriously degrade the signal gradients of such images. Moreover, the degree of the degradation is usually different, depending on the intensity level. Because of the differences of gradient degradations, a pixel in a homogeneous region may have the same gradient value as one in a non-homogeneous region, and consequently these pixels are found in the same bin of the gradient histogram of the image. Thus, the basic method of the thresholds generation described in Section II.A is applicable only if the pixels of the image are grouped to make the pixels of different classes, homogeneous and non-homogeneous, in the same group "distinguishable" by their gradients.

The gradient degradation in HDR images is often graylevel dependent. One can divide the gray level range into a number of sub-ranges and assume that, in each of the subranges, the gradient degradation is approximately "linear" and the gray level homogeneity can thus be detected by simply comparing the gradient values of the pixels within the subrange with the thresholds generated in it. Fig. 3 illustrates the process of the pixel grouping, classification and mask generation, if the image gray level range is divided into the high, medium and low sub-ranges. The pixels in the gradient map are sorted into three groups, $I_{g 1}, I_{g 2}$ and $I_{g 3}$, according to their initial gray levels, resulting in the three gradient distributions presented in Fig. 4. By means of the pixel grouping, the global gradient distribution is decomposed, and the pixels in the same gradient level are to be classified differently according to their initial gray levels.

In the process shown in Fig. 3, in the first block of "Classification 1", the basic method presented in Section II.A is applied to the pixel group $I_{g l}$ to generate the first three thresholds, i.e., $T h_{L l}, T h_{M l}$ and $T h_{H 1}$, and then the three binary masks, $B_{L l}, B_{M l}$, and $B_{H l}$, from the same gradient pixel group $I_{g 1}$. Similarly, the other two sets of masks, namely $\left(B_{L 2}, B_{M 2}\right.$, $\left.B_{H 2}\right)$ and $\left(B_{L 3}, B_{M 3}, B_{H 3}\right)$, are generated by the two other "Classification 1" blocks. In the block of "Combination", $B_{H l}$, $B_{H 2}$ and $B_{H 3}$ are combined to make Mask 1 that is to shield non-homogeneous pixels from the low-pass filtering. Mask 2 is made of $B_{M 1}, B_{M 2}$ and $B_{M 3}$, and Mask 3 of $B_{L 1}, B_{L 2}$ and $B_{L 3}$.

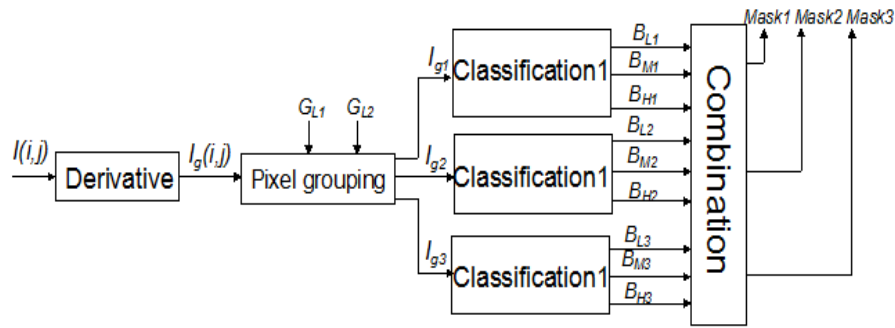

Fig. 3 Block diagram of the classification algorithm with pixel grouping based on gray level ranges.
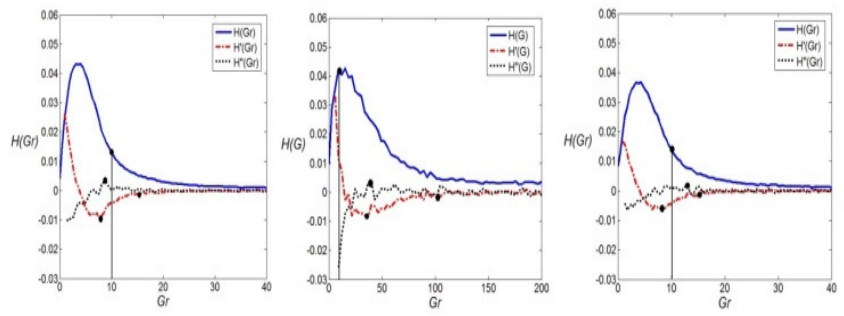

Fig. 4 Gradient distributions given by $I_{g l}, I_{g 2}$ and $I_{g 3}$ pixel groups. Each is normalized with the group pixel population. 
One can see that, by means of the pixel grouping, the global gradient distribution is decomposed into those given by the pixel groups. The pixels in the same bin of the former are sorted, put into the three bins in the three gradient histograms, respectively, and then classified differently according to their gray level sub-range. A better classification result should be expected.

\section{Classification algorithm with pixel grouping by histogram-thresholding}

The gradient degradation in a low contrast image may not necessarily be gray-level-dependent. The algorithm presented in this sub-section is designed for more general cases. In this algorithm, the pixels in the input image are grouped according to their likeness to be in homogeneous (or non-homogeneous) regions. Histogram thresholding [8] is used for this purpose. Fig. 5(a) illustrates a gray level histogram curve of an image. Its high peaks mainly formed by homogeneous pixels in the image. The pixels in the dashed bins are grouped to make the high-bin group, and the remaining pixels form the other group, referred to as low-bin group. Most pixels in the high-bin group are homogeneous pixels, but there is a minority of nonhomogeneous pixels having identifiably higher gradients. Pixels in the "medium-range" of the gradient histogram of this high-bin group are more probably homogeneous. In the lowbin group, the majority is of non-homogeneous pixels, including those of "medium" gradient range of the group, and the minority non-homogeneous pixels are in the upper gradient range. This grouping also results in a decomposition of the global gradient distribution into the two group-gradientdistributions, as shown in Fig. 5(b), in order to differentiate the gradients of the minority pixels from the majority ones in each of the groups. The method presented in Section II.A can then be applied to identify the minority pixels in each group.
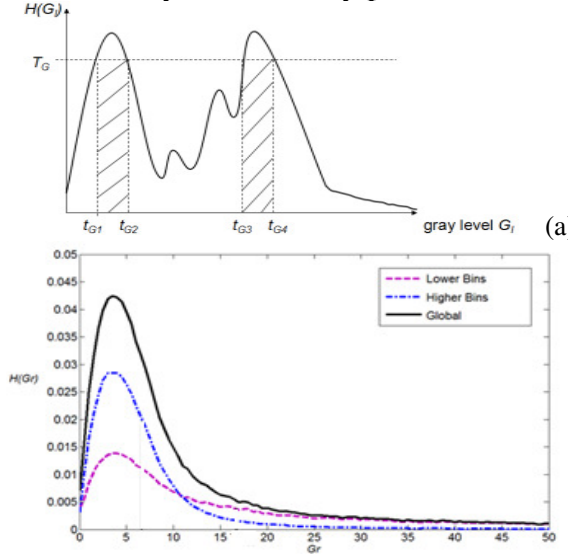

Fig. 5 (a) Example of image gray level histogram.

(b) Example of gradient histograms. The black solid curve is given by the pixels of an image and the other two by its two pixel groups.

Fig. 6 illustrates a block diagram of the algorithm involving the pixel grouping described above, and detailed diagrams of "Pixel grouping" and "Classification 2" blocks are presented in Fig. 7. Three histogram thresholds, $T_{G l}, T_{G 2}$, and $T_{G 3}$, are applied, one by one, to the same gray level histogram of the input image. Each time a binary image, e.g.,
$B_{1}$, is generated to indicate the pixel positions of the high-bin group with one logic value and those of the low-bin one with the other. By using this binary image $B_{l}$, the gradient map $I_{g}$ is divided, in "Pixel grouping" block, into the high-bin and lowbin groups, namely $I_{g l-0}$ and $I_{g l-l}$. In each block of "Classification 2", a gradient histogram analysis presented in Section II.A is applied to each of the two gradient pixel groups, but the procedure is simplified as only one threshold is needed from each group. As shown in Fig. 7. $T h_{H}$ is generated from the gradient histogram of the high-bin group and $T h_{L}$ from the low-bin group. The two thresholds are then used to classify the pixels of the image in such a way that, if a pixel gradient value $G_{r}$ is between $T h_{L}$ and $T h_{H}$, the pixel will be classified as homogeneous one if it is in the high-bin group, otherwise as non-homogeneous one.

The histogram threshold $T_{G}$ can be adjusted to determine the concentration of the majority homogeneous pixels in the high-bin group and that of the majority non-homogeneous pixels in the low-bin one. Among $T_{G 1}, T_{G 2}$ and $T_{G 3}$ shown in Fig. 6, $T_{G l}$ is the lowest, and it makes the low-bin group $I_{g l-1}$ have a good concentration of non-homogeneous pixels, i.e., a good sorting for the non-homogeneous ones and putting all the remaining pixels in the high-bin group $I_{g 1-0}$. The subsequently generated Mask 1 is used to distinguish the non-homogeneous pixel positions from the rest of the image. Similarly, $T_{G 3}$, the highest threshold, leads to the generation of Mask 3 that exposes the homogeneous pixel positions and shields all the others.

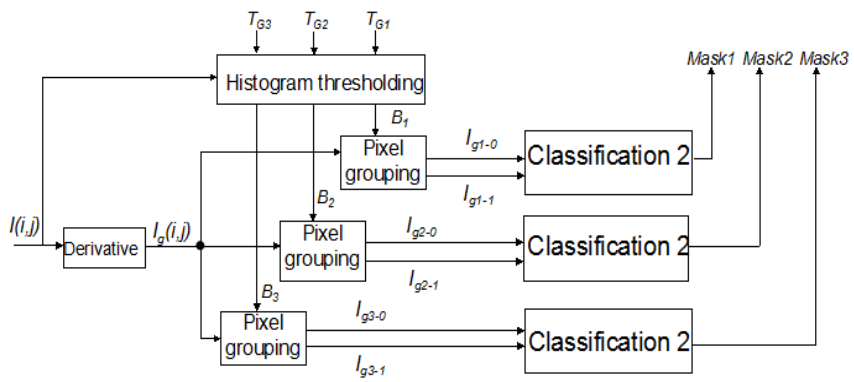

Fig. 6 Block diagram of the classification algorithm with pixel grouping by histogram-thresholding.

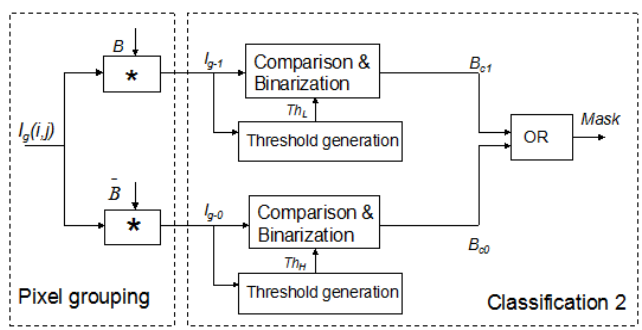

Fig. 7 Detailed diagram of the blocks of Pixel grouping and Classification 2 shown in Fig. 6.

\section{Simulation RESUltS}

The two pixel classification algorithms proposed in the Sections II have been incorporated into the contrast enhancement scheme shown in Fig. 1. The simulations of the scheme have been done to evaluate the effectiveness of the classification algorithms in the noise removal and signal preservation for a high-quality contrast enhancement. CLAHE 
process with a tile size of $8 \times 8$ and clipping limit of 0.03 is used in the block of "contrast enhancement". Four cascaded Gaussian LP filters are used and the first one, i.e., the prefilter, has its window sized $3 \times 3$ pixels and $\sigma=0.5$. The other 3 filters are identical, each having $5 \times 5$ windows and $\sigma=1.0$ to provide a moderate smoothing operation. The four SOBEL kernels, detecting the gray level variations in the horizontal, vertical and two diagonal directions, are used in the HP block to obtain the weighted gradients. For the algorithm proposed in Section II.B, the gray level sub-ranges are $(0,100),(101$, $200)$ and $(201,255)$. In case of the other proposed algorithm, $T_{G I}, T_{G 2}$, and $T_{G 3}$ are $10 \%, 30 \%$ and $80 \%$ of the maximum binheight in the gray level histogram. The simulation results are compared with those obtained by the two high-quality contrast enhancement algorithms, i.e., the iterated TMR filtering [5] and the adaptive histogram clipping [3]. Both algorithms aim at reducing noise generated in the CLAHE process.

Comparing the processed images presented in Fig. 8(b)(f), one can see that (i) while enhancing significantly the image contrast, the CLAHE generates visible noise, particularly in the lower part of the image, (ii) some image details are lost in (c), and (iii) the processed images in (e) and (f) have more image details and less noise.

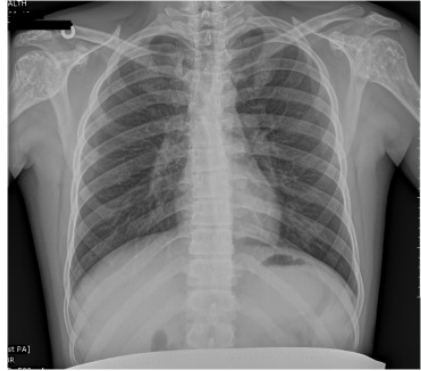

(a)

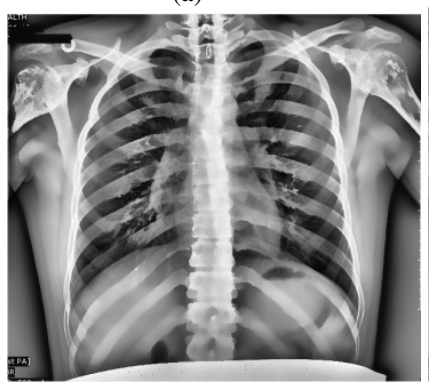

(c)

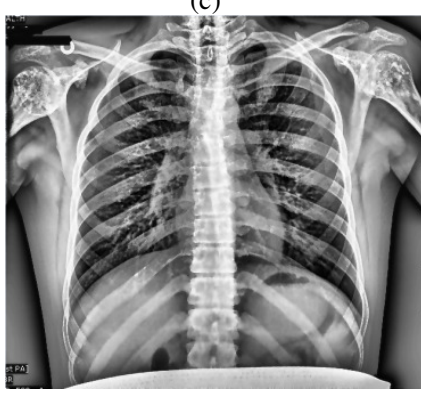

(e)

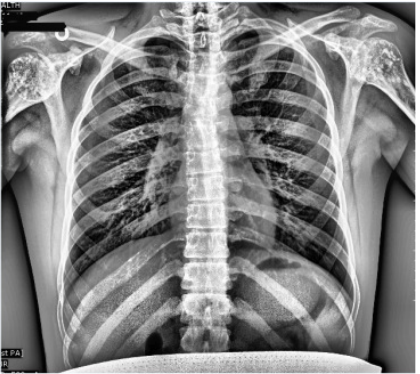

(b)

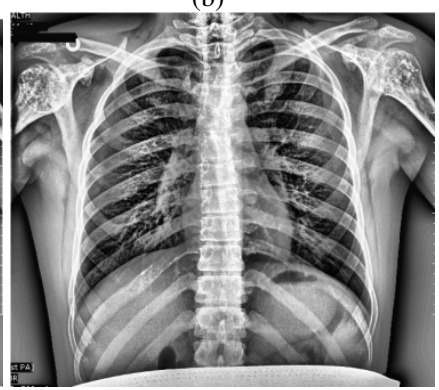

(d)

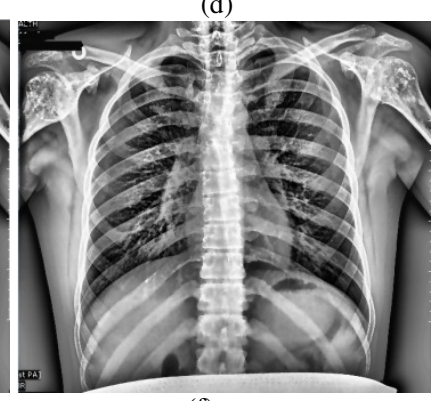

(f)

Fig. 8 (a) Input X-rays image, and the results given by (b) the CLAHE, (c) the iterated TMR[5], (d) the adaptive clipping [3], (e) the algorithm proposed in $\S$ II.B, and (f) that in $\S$ II.C.
The images in Figs. 9 and 10 contain a lot of fine details, corresponding to high spatial frequency components. In order to better illustrate such details, only a segment of each processed image is shown. In the images processed by the CLAHE, noise is very visible around the boundaries of fine objects, for instance, pieces of pollen grains in Fig. 9(b), or the pens in the left side of Fig. 10(b). By comparing the processed images shown in Figs. 9 and 10, one can see that the proposed algorithms yield a better noise reduction in the areas of fine objects, without erasing the image details, with respect to those of the iterated TMR filtering and the adaptive histogram clipping.

The PSNR and the Pratt's Figure of Merit (PFOM) [9] of the processed images of Window \& Desk and Pollen Grain have been measured. The results are presented in Table 1. In case of Window \& Desk, the two proposed algorithms yielded better results combining PSNR and FOM. In case of Pollen and Grain, all the four algorithms produced similar values of PSNR and FOM, maybe due to the relatively low-quality of the available reference image used in the measurement.

\begin{tabular}{|c|c|c|c|c|c|c|c|c|}
\hline \multirow{2}{*}{$\begin{array}{l}\text { Method } \\
\text { Input } \\
\text { image }\end{array}$} & \multicolumn{2}{|c|}{ Iterated TMR filtering } & \multicolumn{2}{|c|}{ Adaptive clipping } & \multicolumn{2}{|c|}{ Algorithm in $\$ 2.2$} & \multicolumn{2}{|c|}{ Algorithm in $\$ 2.3$} \\
\hline & PSNR & PFOM & PSNR & PFOM & PSNR & PFOM & PSNR & PFOM \\
\hline $\begin{array}{l}\text { Window } \\
\text { and Desk }\end{array}$ & 18.347 & 0.895 & 18.438 & 0.899 & 18.472 & 0.934 & 18.377 & 0.926 \\
\hline $\begin{array}{l}\text { Pollen } \\
\text { Grain }\end{array}$ & 12.075 & 0.945 & 12.024 & 0.960 & 12.078 & 0.964 & 12.077 & 0.970 \\
\hline
\end{tabular}

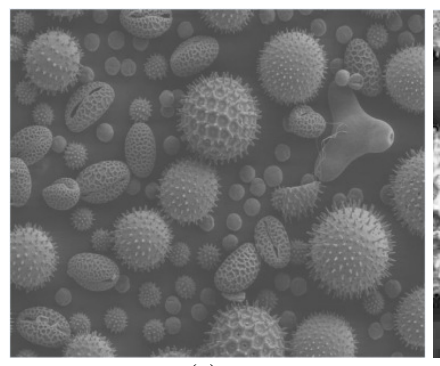

(a)

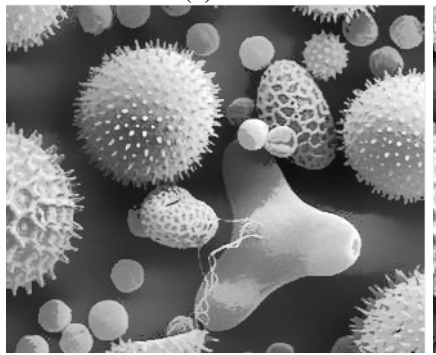

(c)

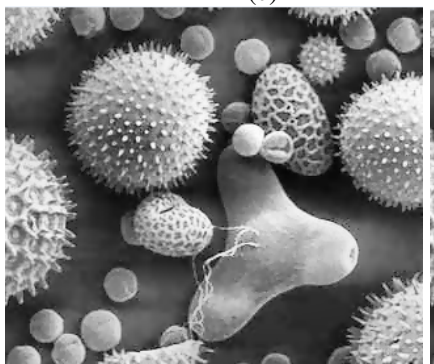

(e)

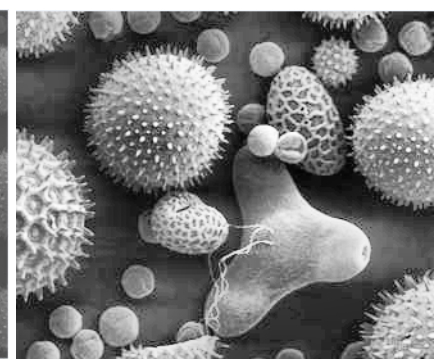

(b)

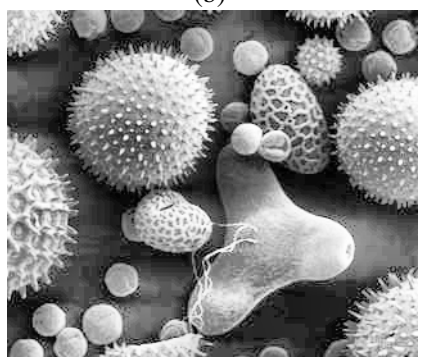

(d)

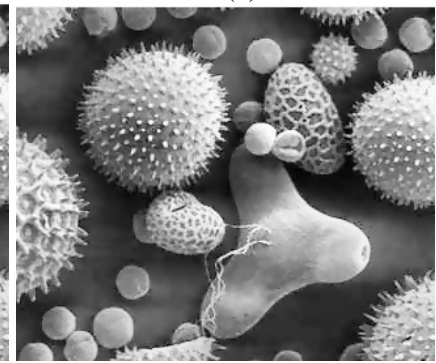

(f)

Fig. 9 (a) Input image of Pollen Grain, and a segment of the processed image given by (b) the CLAHE, (c) the iterated TMR[5], (d) the adaptive clipping [3], (e) the algorithm proposed in § II.B, and (f) that in § II.C. 


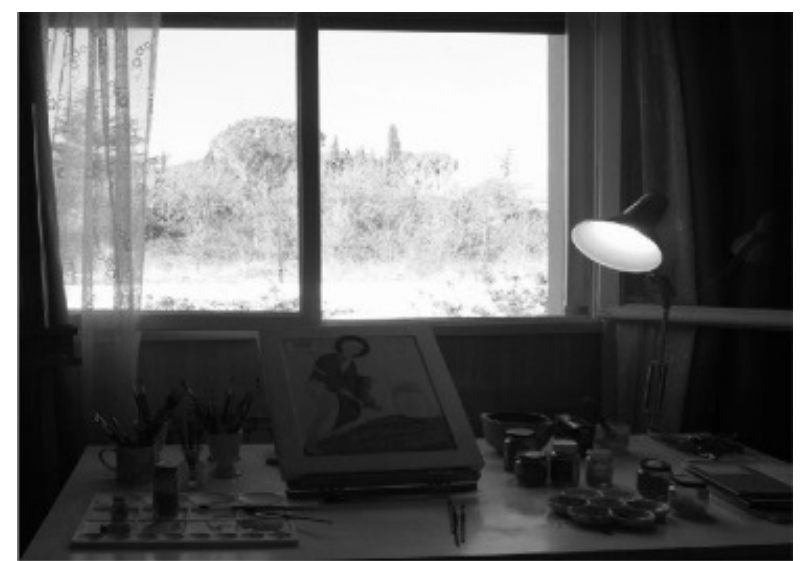

(a)

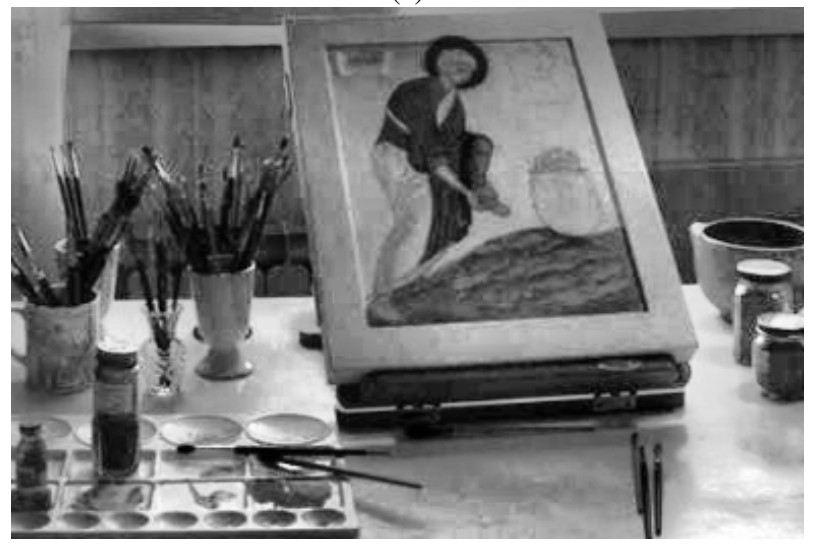

(b)

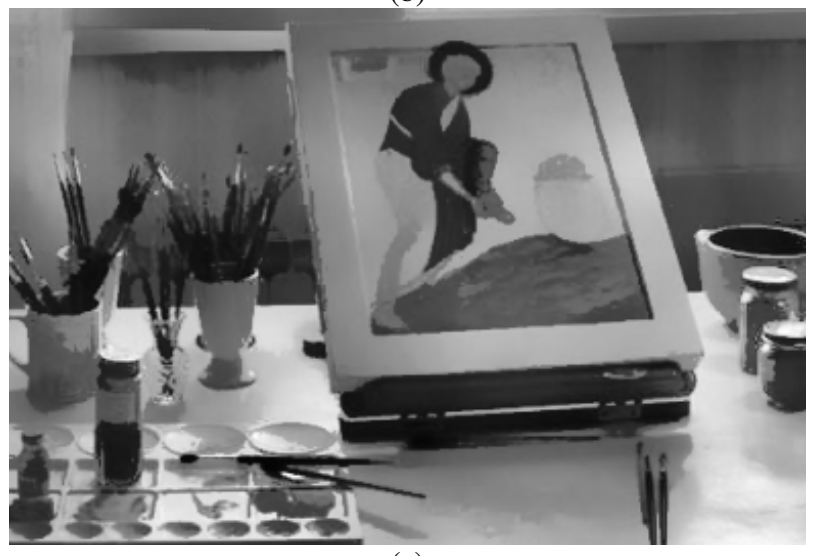

(c)

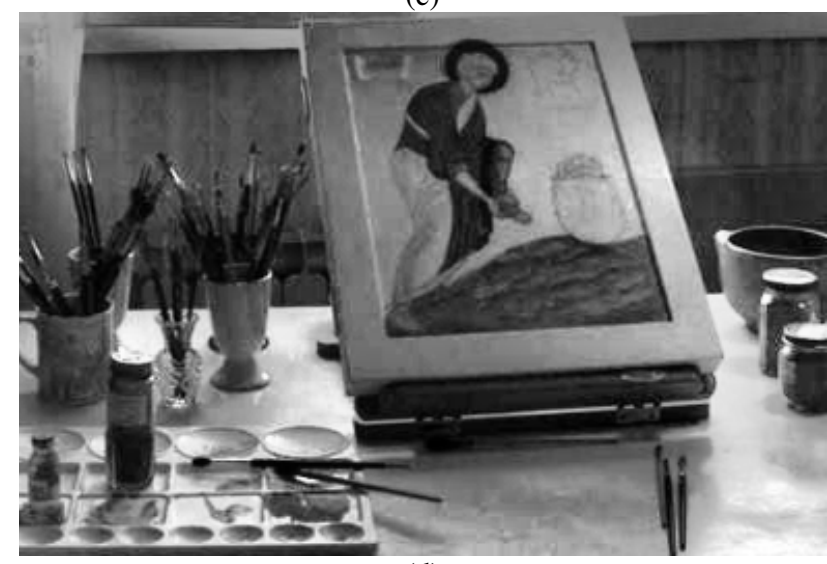

(d)

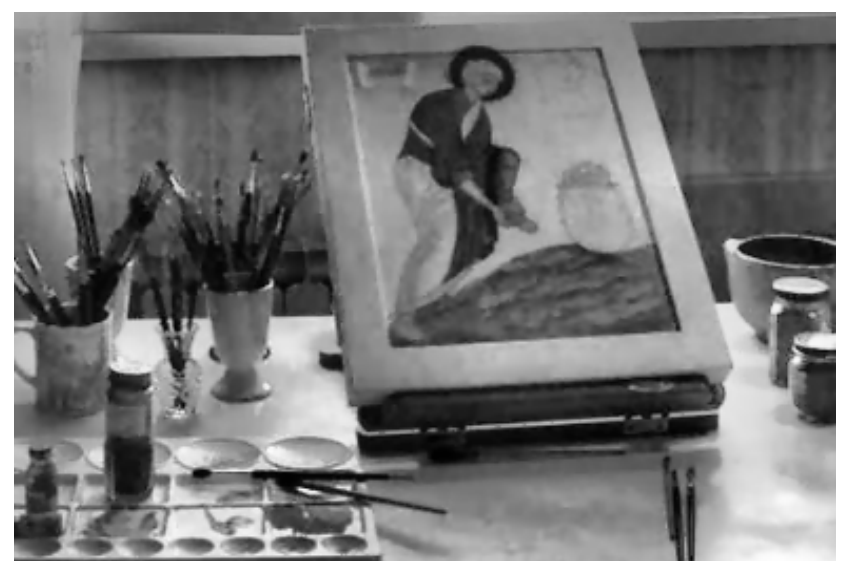

(e)

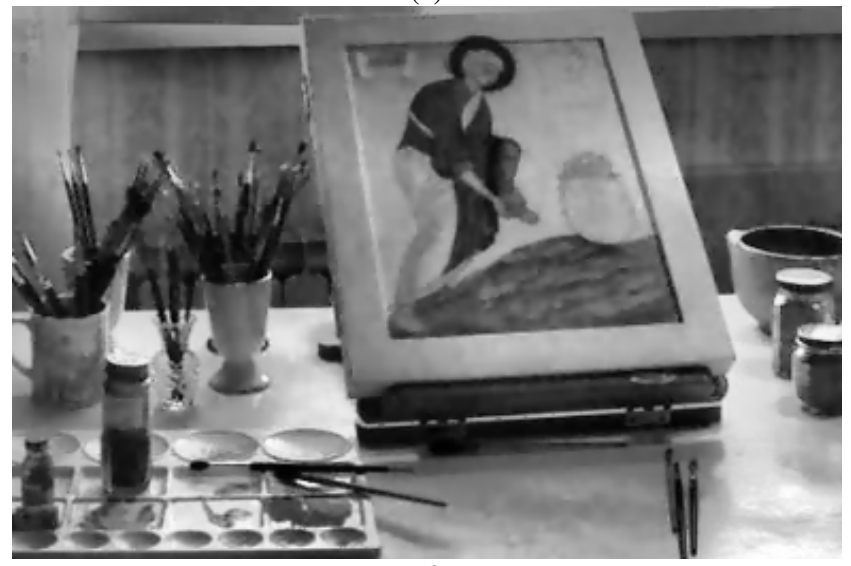

(f)

Fig. 10 (a) Window and Desk, and a segment of the processed image given by (b) the CLAHE, (c) the iterated TMR[5], (d) the adaptive clipping [3], (e) the algorithm proposed in § II.B, and (f) that in § II.C.

\section{CONCLUSION}

In this paper, two algorithms have been proposed to classify the pixels of an image of very poor contrast, according to the homogeneity of their regions. In these algorithms, simple high pass filters are used to obtain the gradient histogram. The classification is based on an analysis of the gradient distribution. To tackle the problems of various gradient degradation patterns in low-contrast images, the gradient histogram of the input image are decomposed so that the pixels having the same gradients but different kinds of neighborhood conditions can be classified differently by applying different gradient thresholds to the decomposed gradient histograms. The decomposition is implemented by pixel grouping. One of the proposed algorithms aims at lowcontrast HDR images, where the image gradients are graylevel dependent, and the pixels are grouped according to their initial gray level ranges. The other algorithm is designed for a wider range of poor-contrast images and the pixels are grouped by gray-level-thresholding, i.e. dividing the pixels of the image into gray-level-histogram high-bin and low-bin groups. The majority pixels in the high-bin group are likely located in homogeneous regions and the minority nonhomogeneous pixels in this group can be easily identified by gradient thresholding. In a similar manner, one can easily 
distinguish the minority homogeneous pixels in the low-bin group from the majority non-homogeneous pixels. In case of very complex gradient degradations, one can combine the two pixel grouping methods, e.g. grouping the pixels in a sub gray level range into high-bin and low-bin groups, to make the decomposition of the gradient distribution more precise for a better quality of the pixel classification.

The proposed algorithms can be applied in a process of noise removal, or for high-quality edge detections. Each of the algorithms has been incorporated into a procedure of lownoise contrast enhancement. It has been proven by simulation that the binary masks resulting from the classification can make the low-pass filters effectively remove the noise generated in the histogram equalization while well preserving the signal details.

\section{REFERENCES}

[1] S.M. Pizer, E.P. Amburn, J.D. Austin, R. Cromartie, A. Geselowitz, T. Greer, B. Ter Haar Romeny, J.B. Zimmerman, and K. Zuiderveld, "Adaptive Histogram Equalization and Its Variations," Computer Vision, Graphics, and Image Processing, vol.39, pp. 355-368, 1987.

[2] J. Stark, "Adaptive Image Contrast Enhancement using Generalizations of Histogram Equalization," IEEE Transactions on Image Processing, vol.9, No.5, pp.889-896, 2000.

[3] A. Boschetti, N. Adami, R. Leonardi, and M. Okuda, "High Dynamic Range Image Tone Mapping Based on Local Histogram Equalization," IEEE International Conference on Multimedia \& Expo, 2010.

[4] J.-Y. Kim, L.-S. Kim and S.-H. Hwang, "An Advanced Contrast Enhancement Using Partially Overlapped Sub-block Histogram Equalization," IEEE Transactions on Circuits and Systems for Video Technology, vol.11, No.4, pp.475-484, 2001.

[5] J. Rabin, J. Delon, and Y. Gousseau, "Removing Artefacts from Color and Contrast Modifications," IEEE Transaction on Image Processing, vol.20, No.11, pp.3073-3085, 2011.

[6] C. Wang and B. Nahar, "Low-Pass Filtering Aiming at Noise Generated in a Contrast Enhancement," IEEE Midwest Symposium on Circuits and Systems, Ohio, USA, August 2013, pp. 1180-1183.

[7] T.-L. Ji, M. K. Sundareshan, and H. Roehrig, "Adaptive Image Contrast Enhancement Based on Human Visual Properties," IEEE transactions on medical imaging, vol.13, No.4, pp.573-586, 1994.

[8] P. Sahoo, S. Soltani and A. Wong, "A survey of Thresholding Techniques," Computer Vision, Graphics, and Image Processing, vol.41, No.2, pp.233-260, 1988.

[9] William K. Pratt, Digital Image Processing: PIKS Inside, Third Edition. John Wiley \& Sons, Inc. 2001. 